\title{
(6) OPEN ACCESS \\ Optical coherence tomography shows retinal abnormalities associated with optic nerve disease
}

\author{
Kirstin L Tawse, Thomas R HedgesIII, Marisa Gobuty, Carlos Mendoza-Santiesteban
}

New England Eye Center, Tufts Medical Center, Tufts University, Boston, Massachusetts, USA

Received 23 September 2013 Revised 14 January 2014 Accepted 17 February 2014 Published Online First 13 March 2014

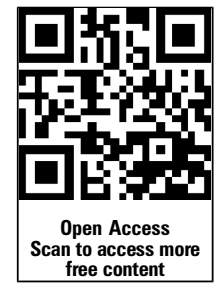

\begin{abstract}
Optical coherence tomography (OCT) of the macula in patients with primary optic neuropathy has revealed the presence of structural changes in the neurosensory retina in addition to the nerve fibre layer. Subretinal fluid has been documented in papilloedema and non-arteritic ischaemic optic neuropathy, and may account for decreased visual acuity in affected patients. Subretinal fluid has also been described from other causes of optic nerve head swelling including diabetic papillopathy and papillitis. Drugs used in the treatment of multiple sclerosis, such as corticosteroids and fingolimod can cause decreased vision due to central serous and cystoid macular oedema sometimes confused with recurrent optic neuritis. A subset of patients with various types of optic atrophy show microcystic changes in the inner nuclear layer on spectral domain OCT imaging. The pathophysiology and visual significance of these retinal changes remain unclear, but may affect the diagnosis and management of optic nerve disorders.
\end{abstract}

\section{INTRODUCTION}

Optical coherence tomography (OCT) is a wellestablished technique that has allowed qualitative and quantitative description of optic nerve disease with a degree of resolution and precision not previously afforded by clinical examination alone. Since OCT was first applied clinically at the New England Eye Center in the early 1990s, we have demonstrated structural changes in the retina and nerve in a variety of optic nerve disorders. As OCT imaging becomes part of the standard of care, a more thorough evaluation and understanding of these structural changes may aid in disease management.

\section{SUBRETINAL FLUID IN PAPILLOEDEMA}

Papilloedema mainly causes visual field loss from damage to the retina nerve fibre layer. Macular changes from papilloedema have been described since $1869^{1}$ but have generally been viewed as secondary phenomena. Morris and Sanders described preretinal and subretinal haemorrhages, choroidal folds, macular exudates and retinal pigment epithelial changes associated with papilloedema. They proposed that a combination of haemodynamic and mechanical factors in papilloedema produced these changes which, in some cases, results in secondary visual loss. ${ }^{1}$ Gittinger and Asdourian hypothesised that mottled macular pigmentation observed in association with papilloedema may be due to macular oedema, similar to the pigmentary changes seen in cystoid macular oedema. ${ }^{2}$ Corbett $e t \mathrm{al}^{3}$ described subretinal fluid in the peripapillary region in cases of papilloedema, causing enlargement of the blindspot, peripapillary hyperopia and refractive scotomas.

In 2001, we demonstrated the presence of subretinal macular oedema on OCT in patients with papilloedema and reduced visual acuity. ${ }^{4}$ Of 19 patients with macular OCT imaging during periods of acute, subacute, or recurrent papilloedema, seven patients had subretinal fluid involving the macula, all of whom had some reduction in visual acuity. Of the 12 patients without subretinal fluid, visual acuities were better than or equal to 20/20 in all but one eye in one patient (in which there was an altitudinal field defect). ${ }^{4}$ All the patients found to have macular subretinal fluid had improvement in central vision as the fluid resolved. ${ }^{4}$ Since then, we have seen this in about $10 \%$ of affected patients (figure 1). In some of these cases, a direct communication between the swollen optic nerve and the submacular space is identified, suggesting that the fluid may originate from the optic nerve. This idea is supported by Samuels's histopathologic findings in 1938 suggesting that the intermediary tissue of Kuhnt can become disrupted with optic disc swelling and may allow escape of peripapillary fluid from the peripapillary choroid. ${ }^{5}$

Savini et $a l^{6}{ }^{7}$ also observed the presence of a hyporeflective subretinal space on OCT in 12 eyes with various types of optic disc oedema, and hypothesised that in addition to the previously proposed idea of peripapillary transudation and exudation of fluid into the subretinal space, extensive swelling of the optic nerve head may anteriorly displace the peripapillary nerve fibre layer producing a tractional separation between the sensory retina and the retinal pigment epithelium. Johnson $e t a l^{8}$ also documented the presence of a hyporeflective space on OCT imaging underlying the sensory retina which had differing characteristics in pathologic disc swelling compared with optic nerve head drusen. They found that this space was on average thicker and more likely to have a smooth internal contour with tapering ' $\mathrm{V}$ ' pattern away from the nerve in cases of pathologic optic disc swelling in comparison to cases of optic disc drusen where the space was, on average, thinner with a more rounded internal contour. They postulated that the most plausible cause for this space was the extravasation of fluid from the optic nerve head percolating into and elevating the subretinal space. It was felt that, due to its longstanding nature, the subretinal fluid in optic nerve head drusen was limited by a homeostatic balance achieved between the capillary and interstitial tissue hydrostatic pressures, and the active transport of the retinal pigment epithelium. However, in cases of recent-onset acute 


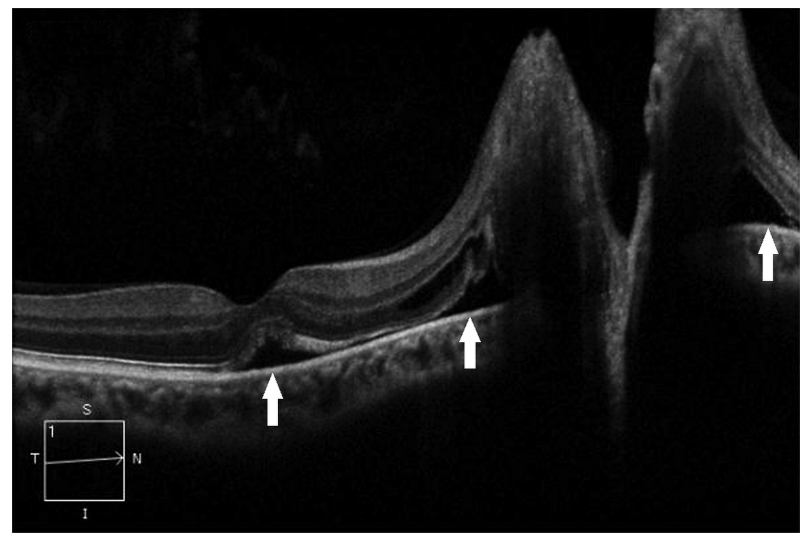

Figure 1 Spectral domain optical coherence tomography line scan from five-line raster protocol through the optic disc and macula showing peripapillary and submacular fluid (arrows) in a middle-aged patient with blurred vision, headaches and nosebleeds. The patient had papilloedema associated with Waldenström's macroglobulinemia.

pathologic disc oedema, a relatively sudden increase in capillary hydrostatic pressure was thought to allow for volumetric expansion of the subretinal fluid and extension into the submacular space in some cases as previously described. ${ }^{8}$ Finally, Scott $e t$ al ${ }^{9}$ proposed that the hyporeflective subretinal space seen on OCT in papilloedema does not necessarily represent fluid in all cases, but rather represents artefact secondary to a thickened and more reflective retinal nerve fibre layer which they described in cases of papilloedema. ${ }^{7}$

Kupersmith et $a l^{10}$ used OCT to quantify deformation of the peripapillary subretinal structures and found that peripapillary retinal pigment epithelium and Bruch's membrane at the optic canal opening was more commonly deflected inward towards the vitreous in cases of papilloedema than in eyes with nonarteritic anterior ischaemic optic neuropathy (NAION) or optic neuritis. They felt these changes were secondary to an elevated pressure gradient between the retrolaminar subarachnoid perioptic nerve sheath compartment and the globe creating a mechanical deformation of the peripapillary subretinal structures. The inward angulation of the retinal pigment epithelium and Bruch's membrane was noted to resolve as papilloedema subsided.

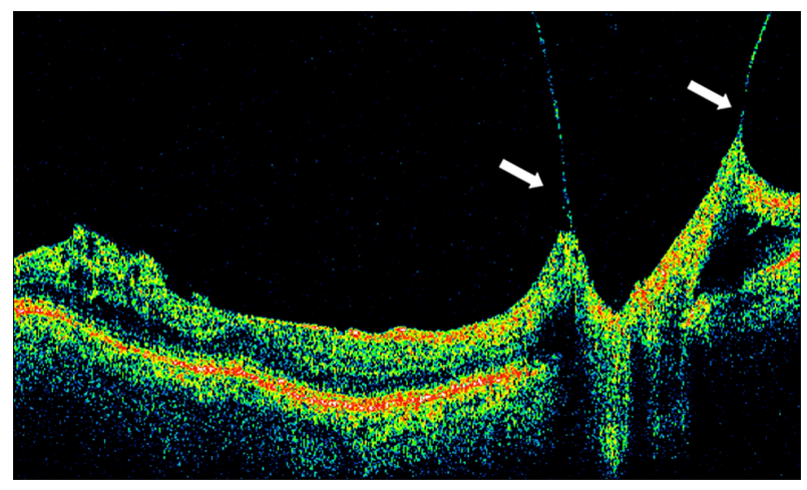

Figure 2 Time domain optical coherence tomography line scan through the optic nerve and macula showing vitreous adhesions (arrows) apparently exerting anterior traction on the optic disc margins in an elderly patient who was referred for apparent optic nerve head swelling in the right eye.

\section{VITREOPAPILLARY TRACTION}

In 2006, we described two cases referred for evaluation of papilloedema in which OCT imaging demonstrated vitreopapillary traction with elevation of the optic nerve head and subsequent blurring of the disc margins with a subretinal hyporeflective space. ${ }^{11}$ However, these patients did not have true disc oedema, but rather the appearance of optic disc swelling created by tractional forces imposed by the contracting vitreous (figure 2). In one case, vitrectomy allowed the optic disc to return to normal.

\section{DIABETIC PAPILLOPATHY AND PAPILLITIS}

Nakamura et al ${ }^{12}$ described cases of serous macular detachment identified on OCT imaging in cases of diabetic papillopathy with minimal or no concurrent diabetic retinopathy. Based on fluorescein angiogram findings in these cases, they too concluded that the fluid resulted from leakage from the optic nerve head rather than a disruption of the blood-retinal barrier. Furthermore, they proposed that diabetes mellitus-induced glial dysfunction may accelerate transretinal fluid movement in such cases. ${ }^{12}$ We have also seen subretinal fluid on OCT in cases of papillitis associated with meningitis with improvement in visual acuity following resolution of the serous retinal detachment. Also, there appeared to be more hyper-reflective material below a clear area suggesting subretinal inflammation (figure 3).

\section{SUBRETINAL FLUID IN ISCHEMIC OPTIC NEUROPATHY}

In 2008, we described the presence of submacular fluid in patients with NAION. ${ }^{13}$ Of 76 patients with NAION from two institutions, 8 patients had subfoveal fluid on OCT, while 28 of the 44 patients from the New England Eye Center had peripapillary fluid and/or subretinal fluid extending towards but not into the fovea. Visual acuity reduction roughly correlated with the degree of increased macular thickness. It was proposed that subretinal fluid may contribute to some of the visual loss associated with anterior ischaemic optic neuropathy, and that resolution of the fluid may account for a portion of the visual improvement that often occurs with NAION. A fluorescein angiogram done in one of these patients did not show accumulation of dye in the macular region, indicating the fluid did not arise from the retinal vessels or directly from the choroid. Furthermore, it was suggested that there may be disruption of the glial tissues that comprise the intermediary tissue of Kuhnt, similar to what occurs in papilloedema. Subretinal fluid is allowed to escape from the peripapillary choroid into the subretinal space and track into the macula ${ }^{13}$ (figure 4). Visual acuity improved in five of eight patients described, as the subfoveal fluid resolved. In that study and in subsequent observations, macular fluid occurs in about $15 \%$ of patients with anterior ischaemic optic neuropathy. As our treatment of optic neuropathies evolves and new treatments become available, it becomes increasingly important to understand specifically what is being treated. Indeed, decreased vision due to associated subretinal fluid may be treated differently than visual acuity changes arising from the optic neuropathy itself.

\section{DRUG-INDUCED RETINOPATHY}

Secondary retinopathy in the setting of treatment of primary optic nerve disease is also well described. Corticosteroids are routinely used in the treatment of optic neuritis and recurrent demyelinating attacks in multiple sclerosis, but are also known to be associated with development of central serous retinopathy. Patients who have multiple sclerosis and loss of vision may be 

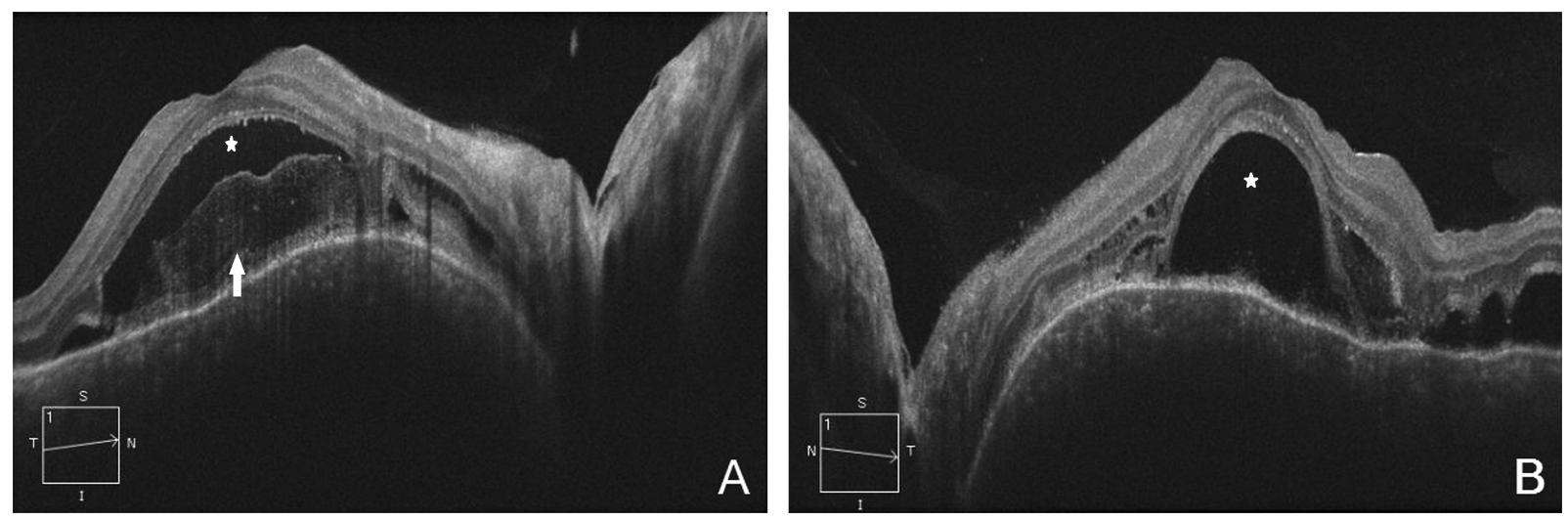

Figure 3 (A and B): Spectral domain optical coherence tomography line scan of the right eye (A) and left eye (B) showing subretinal fluid (stars) in a middle-aged patient with headaches and decreased central vision from bilateral papillitis and aseptic meningitis with normal intracranial pressure. The increased reflectivity in the subretinal space in the right eye possibly represents inflammatory exudate (arrow). The patient had clinical and anatomic improvement with corticosteroids.

misdiagnosed with optic neuritis when they actually have central serous retinopathy, making retinal evaluation of particular importance in patients with multiple sclerosis who routinely receive steroids as part of their disease management. Additionally, although neuro-ophthalmic evaluation for associated optic neuritis is often part of multiple sclerosis disease management, the ophthalmologist must be careful to not neglect associated vasculitis or uveitis that may also cause retinal abnormalities including cystoid macular oedema and a subsequent reduction in visual acuity.

Fingolimod has recently been approved as an oral treatment option for multiple sclerosis, and two phase III clinical studies demonstrated a significant reduction in the relapse rate in patients with relapsing-remitting multiple sclerosis compared with interferon and placebo. ${ }^{14} 15$ However, macular oedema was an adverse event reported in these and prior studies. Central foveal thickness measurements on OCT in such cases are often increased, and cross-sectional analysis demonstrates hyporeflective pockets or cysts within the retina, and an altered contour of the retina. ${ }^{16}$ Fingolimod is a structural analogue to sphingoine-1-phosphate (S1P) which plays a key role in the release of $\mathrm{T}$ lymphocytes from secondary lymphoid organs,

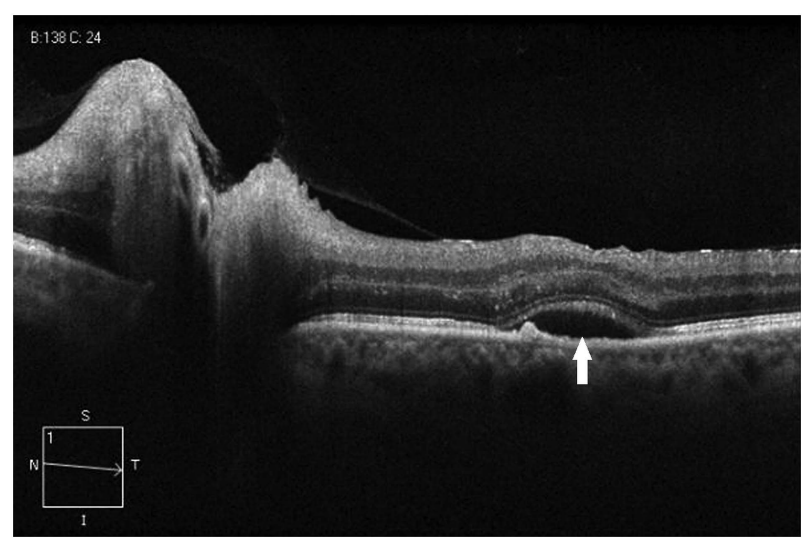

Figure 4 Spectral domain optical coherence tomography line scan through the optic disc and macula showing subretinal fluid (arrow) in a middle-aged patient with a history of insulin-dependent diabetes mellitus, hypertension, hyperlipidaemia and sleep apnoea, who awoke with decreased vision in the left eye due to non-arteritic anterior ischaemic optic neuropathy. thereby decreasing the population of circulating lymphocytes. However, the S1P receptor also plays a role in the regulation of vascular permeability and is implicated in the process of fingolimod-associated macular oedema, a well-described dosedependent side effect more likely to occur in diabetics or patients with a prior history of uveitis. ${ }^{16} 17$

\section{MICROCYSTIC RETINAL CHANGES}

Gelfand et al ${ }^{18}$ described macular microcystic changes predominantly involving the inner nuclear layer of the retina identified on spectral domain OCT images in patients with multiple sclerosis who did not have another reason for macular oedema. It was suggested in this paper that this finding may be a result of inner nuclear inflammation and microglial activation resulting in a breakdown of the blood-retinal barrier. ${ }^{18}$ These microcystic changes in the inner nuclear layer were also found in eyes with a prior history of optic neuritis in a significant portion of patients with neuromyelitis optica. ${ }^{19}$ However, similar microcystic changes in the inner nuclear layer have been recently described in cases of compressive optic neuropathy, optic atrophy due to glaucoma, Leber's hereditary optic atrophy, dominant optic atrophy, trauma and hydrocephalus. ${ }^{20-22} \mathrm{We}$ have recently seen these changes in a child with optic atrophy from hydrocephalus (figure 5). The presence of these changes in inflammatory and non-inflammatory conditions, and the lack of leakage on fluorescein angiography, argue against a breakdown in the blood-retinal barrier. ${ }^{22}$ Vitreous traction has been proposed as a cause of retinal microcystic changes seen in optic atrophy, ${ }^{22}$ although recent studies have reported only cases in which the absence of vitreoretinal traction was confirmed with OCT. $^{21}$ Microcystic changes may be due to trans-synaptic degeneration resulting from optic nerve damage. ${ }^{20}$ This theory is supported by the histopathologic finding of cavitary degeneration in the inner nuclear layer in optic nerve crush experiments in primates by Van Buren in $1963 .{ }^{23}$

\section{COEXISTENT PRIMARY RETINOPATHY}

Independent primary retinopathy in the setting of known optic neuropathy can also coexist, and patients with retinopathy can also develop optic neuropathy. A thorough examination of the retina, with appropriate imaging when indicated, is an important component in the workup and management of all optic neuropathies, and patients with retinopathy should have periodic 

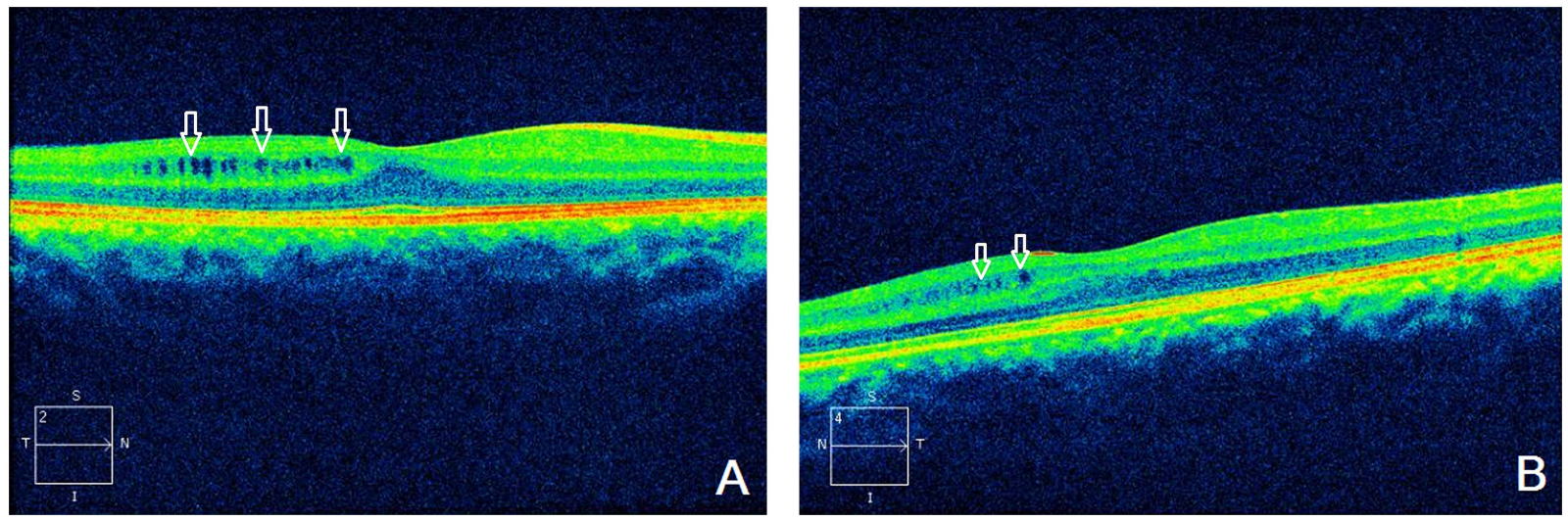

Figure 5 (A and B) Spectral domain optical coherence tomography line scan showing bilateral retinal microcystic changes (arrows) temporally in the right eye (A) and nasal to the fovea in the left eye (B) in a child with optic atrophy secondary to hydrocephalus.

OCT scanning of the retinal nerve fibre layer, especially if vision loss seems out of proportion to the retinal findings.

\section{CONCLUSION}

OCT imaging has expanded our understanding of the pathology of many diseases of the retina and nerve, and has revealed structural changes in both that were previously unknown. OCT has further demonstrated the intimate connection between pathology in the neurosensory retina and optic nerve. Understanding the diagnostic and therapeutic value of these findings will improve the diagnosis and treatment of patients with optic neuropathy.

Contributors All authors contributed to, reviewed and approved the final version of the manuscript. TRH and KLT designed, wrote the first draft, reviewed and edited the manuscript. Guarantors: TRH, KLT.

\section{Competing interests None.}

Provenance and peer review Not commissioned; externally peer reviewed.

Open Access This is an Open Access article distributed in accordance with the Creative Commons Attribution Non Commercial (CC BY-NC 3.0) license, which permits others to distribute, remix, adapt, build upon this work non-commercially, and license their derivative works on different terms, provided the original work is properly cited and the use is non-commercial. See: http://creativecommons.org/ licenses/by-nc/3.0/

\section{REFERENCES}

1 Morris AT, Sanders MD. Macular changes resulting from papilloedema. $\mathrm{Br} J$ Ophthalmol 1980;64;211-16.

2 Gittinger JW, Asdourian GK. Macular abnormalities in papilledema from pseudotumor cerebri. Ophthalmology 1989:96:192-4.

3 Corbett JJ, Jacobson DM, Maur RC, et al. Enlargement of the blind spot caused by papilledema. Am J Ophthalmol 1988;105:261-5

4 Hoye VJ III, Berrocal AM, Hedges TR III, et al. Optical Coherence Tomography Demonstrates Subretinal Macular Edema From Papilledema. Arch Ophthalmol 2001;119:1287-90.

5 Samuels B. Histopathology of papilledema. Am J Ophthalmol 1938;21:1242-8.

6 Savini G, Bellusci C, Carbonelli M, et al. Detection and quantification of retinal nerve fiber layer thickness in optic disc edema using stratus OCT. Arch Ophthalmol 2006;124:1111-7.
7 Savini G, Barboni P, Carbonelli M, et al. A. Optical Coherence Tomography for Optic Disc Edema. Arch Ophthalmol 2011;129:1245-6; author reply 1246-7.

8 Johnson LN, Diehl ML, Hamm CW, et al. Differentiating Optic Disc Edema From Optic Nerve Head Drusen on Optical Coherence Tomography. Arch Ophthalmol 2009:127:45-9.

9 Scott CJ, Kardon RH, Lee AG, et al. Diagnosis and grading of papilledema in patients with raised intracranial pressure using optical coherence tomography vs clinical expert assessment using a clinical staging scale. Arch Ophthalmol 2010;128:705-11.

10 Kupersmith MJ, Sibony P, Madel G, et al. Optical coherence tomography of the swollen optic nerve head: deformation of the peripapillary retinal pigment epithelium layer in papilledema. Invest Ophthalmol Vis Sci 2011;52:6558-64.

11 Hedges TR, Flattem NL, Bagga A. Vitreopapillary traction confirmed by optical coherence tomography. Arch Ophthalmol 2006;124:279-81.

12 Nakamura M, Kanamori A, Nagai-Kusuhara A, et al. Serous macular detachment due to diabetic papillopathy detected using optical coherence tomography. Arch Ophthalmol 2009;127:105-7.

13 Hedges TR, Vuong LN, Gonzalez-Garcia AO, et al. Subretinal fluid from anterior ischemic optic neuropathy demonstrated by optical coherence tomography. Arch Ophthalmol 2008;126:812-15.

14 Cohen JA, Barkhof F, Comi G, et al. Oral fingolimod or intramuscular interferon for relapsing multiple sclerosis. N Engl J Med 2010;362:402-15.

15 Kappos L, Radue EW, O'Connor P, et al. A placebo-controlled trial of oral fingolimod in relapsing multiple sclerosis. N Engl J Me 2010;362:387-401.

16 Jain N, Bhatti TM. Fingolimod-associated macular edema: Incidence, detection, and management. Neurology 2012;78:672-80.

17 Zarbin MA, Jampol LM, Jager RD, et al. Ophthalmic Evaluations in Clinical Studies of Fingolimod (FTY720) in Multiple Sclerosis. Ophthalmology 2013;120:1432-9.

18 Gelfand JM, Nolan R, Schwartz DM, et al. Microcystic macular oedema in multiple sclerosis is associated with disease severity. Brain 2012;135:1786-93.

19 Gelfand JM, Cree BA, Nolan R, et al. Microcystic Inner Nuclear Layer Abnormalities and Neuromyelitis Optica. JAMA Neurol 2013;70:629-33.

20 Abegg $M$, Zinkernagel $M$, Wolf S. Microcystic macular degeneration from optic neuropathy. Brain 2012;135:1-2. (letter to the editor)

21 Wolff B, Basdekidou C, Vasseur V, et al. Retinal inner nuclear layer microcystic changes in optic nerve atrophy: a novel spectral-domain OCT finding. Retina 2013;33:2133-8.

22 Barboni $P$, Carelli V, Savini G, et al. Microcystic macular degeneration from optic neuropathy: not inflammatory, not trans-synaptic degeneration. Brain 2013;136: e239.

23 VanBuren JM. Trans-synaptic retrograde degeneration in the visual system of primates. J Neurol Neurosurg Psychiatry 1963;26;402-9. 